\title{
Continuous-time Look-Ahead Scheduling of Energy Storage in Regulation Markets
}

\author{
Roohallah Khatami \\ University of Utah \\ roohallah.khatami@utah.edu
}

\author{
Masood Parvania \\ University of Utah \\ masood.parvania@utah.edu
}

\author{
Pramod Khargonekar \\ University of California, Irvine \\ pramod.khargonekar@uci.edu
}

\begin{abstract}
Energy storage (ES) devices offer valuable flexibility services, including regulation reserve, in power systems operation that could improve the reliability and cost-efficiency of systems with high penetration of renewable energy resources. In this paper, a continuous-time look-ahead regulation capacity scheduling model is proposed, which more accurately models and schedules the regulation capacity trajectories provided by generating units and ES devices in real-time power systems operation. A function space solution method is proposed to reduce the dimensionality of the continuous-time problem by modeling the parameter and decision trajectories in a function space formed by Bernstein polynomials, which converts the continuous-time problem into a linear programming problem. Numerical results, conducted on the IEEE Reliability Test System, show lower operation cost and less regulation scarcity events in real-time power systems operation due to efficient deployment of the ES flexibility in regulation markets.
\end{abstract}

\section{Introduction}

The large-scale integration of renewable energy resources is adding to the sources of variability and uncertainty in power systems operation and calls for additional flexibility resources to provide increased amounts of ancillary services in the systems operation $[1,2]$. Regulation reserve, which provides fast up and down balancing power and ramping [3], is dispatched by the automatic generation control to counterbalance the variability and uncertainty of net-load (load minus renewable energy). Energy storage (ES) devices represent ideal fast ramping resources to provide additional regulation capacity in markets $[4,5,6,7]$. The recent FERC order 841 mandates the independent system operators (ISOs) and regional transmission operators (RTOs) to remove the barriers ES devices are facing to participate in the capacity, energy, and ancillary service markets in the U.S. [8]. This order aims at rendering the ES devices competitive in the electricity markets and heralds a plethora of opportunities for the precious flexibility resources.

There has been multiple research efforts to develop models for scheduling regulation capacity provided by ES devices. Look-ahead real-time operation models have gained specific attention in this regard as they optimize the operation of resources in real-time operation over a horizon that extends into future times, therefore properly taking into account the intertemporal constraints of ES operation. The look-ahead dispatch of generating units was first proposed in 1980 [9] and is currently implemented by ISOs in their operation processes (see e.g., $[10,11]$ ). A price-based look-ahead scheduling model is proposed in [12] to optimally schedule a joint wind and battery ES system. In [13], the look-ahead ES scheduling is discussed in a centralized real-time microgrid control framework acting through the primary, secondary, and tertiary control levels. Using dynamic uncertainty sets, a robust look-ahead economic dispatch is developed in [14] that co-optimizes the generation of generating units and charging/discharging of ES devices. In [15], using a look-ahead economic dispatch model, the potential of thermal ES devices coupled with renewable energy resources in providing energy and regulation services is explored. Look-ahead bidding strategy of a merchant ES participating in day-ahead energy market is addressed in [16], where the receding scheduling horizon covers two consecutive days and the ES state of charge at the end of the first day is optimized such that maximum benefit is realized in the second day. The regulation reserve offered by the aggregated battery storage of electric vehicles is discussed in [17], where dynamic changes of their mobility behavior, as well as the cost associated with their battery degradation are taken into account.

The current look-ahead ES scheduling models, reviewed above, are based on discrete-time optimization models that model the load and decision trajectories as piecewise constant trajectories that neither appropriately 
capture the faster variations of load happen within the discrete time intervals, nor the fast-ramping capability of resources (including ES devices) to compensate the fast load variations. In addition, the discrete-time models define ramping as the finite difference between the consecutive power schedules, which implies that the resources are forced to ramp linearly from one interval to the next, failing to fully deploy the fast-ramping capability of flexible resources in power systems operation. In order to address this problem, we have developed novel continuous-time scheduling models for power systems operation that integrate higher-fidelity models for the parameter and decision trajectories that are defined over higher-order function spaces formed by Bernstein polynomials [18, 19, 20, 21, 22]. Our current models, however, have focused on energy market applications and have not explored the application in scheduling ancillary services, e.g., regulation.

In this paper, we develop a novel continuous-time look-ahead scheduling model for optimally scheduling the regulation capacity provided by generating units and ES devices in real-time power systems operation. In the proposed model, presented in Section 2, the balancing power provided by generating units are utilized to balance the continuous-time real-time load deviation trajectory of the systems. In addition, the regulation up and down capacity provided by generating units and ES devices are modeled as continuous-time trajectories that are optimally schedule to provide the regulation up and down capacity requirements of the system.

A function space solution method is developed in Section 3 to solve the proposed continuous-time model, which is based on reducing the dimensionality of the decision and parameter trajectories by modeling them in a finite-order function space formed by Bernstein polynomials. The proposed method converts the continuous-time problem into a linear programming problem with the Bernstein coefficients of trajectories as the decision variables. The proposed model is implemented in Section 4 on the IEEE Reliability Test System using the real load data of California ISO, and the conclusions are drawn in Section 5.

\section{Continuous-time Look-Ahead Regulation Scheduling Model}

In this section, we propose a continuous-time look-ahead scheduling model for optimal scheduling of regulation up and down reserve capacity provided by generating units and ES devices in real-time power systems operation. The structure of the proposed model is shown in Fig. 1, where the power system operator receives the bids and constraints of generating units and ES devices to provide regulation up and down reserve,

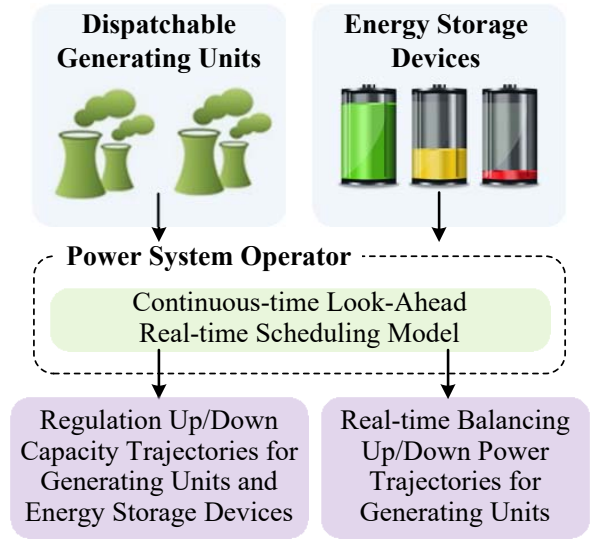

Figure 1. Schematic of the proposed model

and utilizes the proposed look-ahead scheduling model for supplying the real-time load deviation of system and schedule the regulation capacity at minimum cost.

The proposed continuous-time look-ahead scheduling model implements the receding horizon control idea and involves solving an optimal control problem for the next immediate control horizon $\mathcal{T}_{\tau}=[\tau, \tau+T]$, while utilizing the continuous-time state space model of generating units and ES devices. Two consecutive receding control horizons, represented by $\mathcal{T}_{\tau_{1}}$ and $\mathcal{T}_{\tau_{2}}$, are shown in Fig. 2, which start respectively at $\tau_{1}$ and $\tau_{2}$ and end at $\tau_{1}+T$ and $\tau_{2}+T$. The proposed model optimizes the control decisions of generating units and ES devices over each control horizon $\mathcal{T}_{\tau_{1}}, \mathcal{T}_{\tau_{2}}, \ldots$, capturing the intertemporal flexibility and constraints of ES devices and generating units in real-time operation of power systems, implementing only the first instant of control decisions at each run.

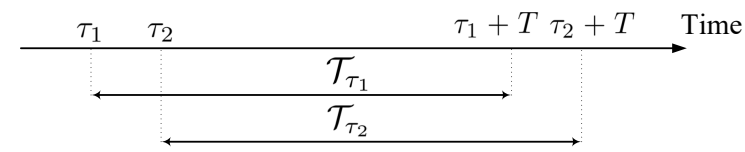

Figure 2. Timeline of the continuous-time look-ahead scheduling model

\subsection{Problem Formulation}

Consider the real-time power system operation problem where a set of $K$ generating units and $J$ energy storage devices are available to balance the real-time load deviation trajectory $\Delta D(t)$ over the receding control horizon $\mathcal{T}_{\tau}$ through providing the up and down balancing power, meanwhile reserving sufficient capacity to meet the continuous-time regulation up and down requirements of the system, $D^{R^{u}}(t)$ and $D^{R^{d}}(t)$. The generating units' real-time up and down 
balancing power trajectories are represented by $\mathbf{g}^{u}(t)=$ $\left(g_{1}^{u}(t), \ldots, g_{K}^{u}(t)\right)^{T}$ and $\mathbf{g}^{d}(t)=\left(g_{1}^{d}(t), \ldots, g_{K}^{d}(t)\right)^{T}$. The associated balancing ramping trajectories are defined as time derivatives of the power trajectories and represented by $\dot{\mathrm{g}}^{u}(t)=\left(\dot{g}_{1}^{u}(t), \ldots, \dot{g}_{K}^{u}(t)\right)^{T}$ and $\dot{\mathrm{g}}^{d}(t)=\left(\dot{g}_{1}^{d}(t), \ldots, \dot{g}_{K}^{d}(t)\right)^{T}$. The regulation up and down services provided by the generating units are represented by $\mathbf{R}^{u, G}(t)=\left(R_{1}^{u, G}(t), \ldots, R_{K}^{u, G}(t)\right)^{T}$ and $\mathbf{R}^{d, G}(t)=\left(R_{1}^{d, G}(t), \ldots, R_{K}^{d, G}(t)\right)^{T}$.

The ES devices can provide regulation up and down capacity respectively through discharging and charging. In charging state, ES devices provide regulation down by drawing power from the grid, and the vector $\mathbf{R}^{d, S}(t)=\left(R_{1}^{d, S}(t), \ldots, R_{J}^{d, S}(t)\right)^{T}$ represents the regulation down trajectories provided by the ES devices. In discharging state, ES devices provide regulation up service by supplying power to the grid, and the vector $\mathbf{R}^{u, S}(t)=\left(R_{1}^{u, S}(t), \ldots, R_{J}^{u, S}(t)\right)^{T}$ denotes the regulation up trajectories.

The continuous-time look-ahead energy generation and storage scheduling problem is formulated here as a variational optimization problem, where the total cost of supplying the real-time load deviation and catering the regulation up and down requirements of the power system over the receding control horizon $\mathcal{T}_{\tau}$ is minimized in the objective functional (1) subject to the operating constraints (2)-(16):

$$
\begin{array}{ll}
\min \int_{\mathcal{T}_{\tau}}\left(\boldsymbol{\rho}^{u} \mathbf{g}^{u}(t)+\boldsymbol{\rho}^{d} \mathbf{g}^{d}(t)+\boldsymbol{\mu}^{u, G} \mathbf{R}^{u, G}(t)+\right. \\
\left.\boldsymbol{\mu}^{d, G} \mathbf{R}^{d, G}(t)+\boldsymbol{\mu}^{u, S} \mathbf{R}^{u, S}(t)+\boldsymbol{\mu}^{d, S} \mathbf{R}^{d, S}(t)\right) d t \\
\Delta D(t)=\mathbf{1}_{K}^{T}\left(\mathbf{g}^{u}(t)-\mathbf{g}^{d}(t)\right), & t \in \mathcal{T}_{\tau}, \\
\mathbf{1}_{K}^{T} \mathbf{R}^{u, G}(t)+\mathbf{1}_{J}^{T} \mathbf{R}^{u, S}(t) \geq D^{R^{u}}(t), & t \in \mathcal{T}_{\tau}, \\
\mathbf{1}_{K}^{T} \mathbf{R}^{d, G}(t)+\mathbf{1}_{J}^{T} \mathbf{R}^{d, S}(t) \geq D^{R^{d}}(t), & t \in \mathcal{T}_{\tau}, \\
\mathbf{G}^{*}(t)+\mathbf{g}^{u}(t)+\mathbf{R}^{u, G}(t) \leq \overline{\mathbf{G}}(t), & t \in \mathcal{T}_{\tau}, \\
\mathbf{G}^{*}(t)-\mathbf{g}^{d}(t)-\mathbf{R}^{d, G}(t) \geq \underline{\mathbf{G}}(t), & t \in \mathcal{T}_{\tau}, \\
\dot{\mathbf{G}}^{*}(t)+\dot{\mathbf{g}}^{u}(t)-\dot{\mathbf{g}}^{d}(t)+\frac{\mathbf{R}^{u, G}(t)}{T^{R}} \leq \overline{\mathbf{G}}(t), & t \in \mathcal{T}_{\tau}, \\
\dot{\mathbf{G}}^{*}(t)+\dot{\mathbf{g}}^{u}(t)-\dot{\mathbf{g}}^{d}(t)-\frac{\mathbf{R}^{d, G}(t)}{T^{R}} \geq \underline{\dot{\mathbf{G}}}(t), & t \in \mathcal{T}_{\tau}, \\
\mathbf{R}^{u, G}(t) & \\
T^{R} &
\end{array}
$$

$$
\begin{aligned}
& \mathbf{0} \leq \mathbf{R}^{u, S}(t) \leq \overline{\mathbf{R}}^{u, S}, \frac{\mathbf{R}^{u, S}(t)}{T^{R}} \leq \overline{\mathbf{R}}^{u, S}, \quad t \in \mathcal{T}_{\tau}, \\
& \mathbf{0} \leq \mathbf{R}^{d, S}(t) \leq \overline{\mathbf{R}}^{d, S}, \frac{\mathbf{R}^{d, S}(t)}{T^{R}} \leq \overline{\mathbf{R}}^{d, S}, \quad t \in \mathcal{T}_{\tau}, \\
& \frac{d \overline{\mathbf{E}^{S}}(t)}{d t}=\boldsymbol{\eta}^{c} \mathbf{R}^{d, S}(t), \quad t \in \mathcal{T}_{\tau}, \\
& \frac{d \mathbf{E}^{S}(t)}{d t}=-\boldsymbol{\eta}^{d^{-1}} \mathbf{R}^{u, S}(t), \quad t \in \mathcal{T}_{\tau} \\
& \underline{\mathbf{E}} \leq \overline{\mathbf{E}^{S}}(t) \leq \overline{\mathbf{E}}, \quad t \in \mathcal{T}_{\tau}, \\
& \underline{\mathbf{E}} \leq \underline{\mathbf{E}}^{S}(t) \leq \overline{\mathbf{E}}, \quad t \in \mathcal{T}_{\tau}, \\
& \mathbf{g}^{u}(\tau)=\mathbf{g}_{\tau}^{u}, \mathbf{g}^{d}(\tau)=\mathbf{g}_{\tau}^{d}, \\
& \mathbf{R}^{u, G}(\tau)=\mathbf{R}_{\tau}^{u, G}, \mathbf{R}^{d, G}(\tau)=\mathbf{R}_{\tau}^{d, G}, \\
& \mathbf{R}^{u, S}(\tau)=\mathbf{R}_{\tau}^{u, S}, \mathbf{R}^{d, S}(\tau)=\mathbf{R}_{\tau}^{d, S}, \\
& \underline{\mathbf{E}^{S}}(\tau)=\overline{\mathbf{E}^{S}}(\tau)=\mathbf{E}_{\tau}^{S} .
\end{aligned}
$$

In the objective functional (1), $\boldsymbol{\rho}^{u, G}$ and $\boldsymbol{\rho}^{d, G}$ are the prices of generating units to provide the up and down balancing service; $\boldsymbol{\mu}^{u, G}, \boldsymbol{\mu}^{d, G}, \boldsymbol{\mu}^{u, S}$ and $\boldsymbol{\mu}^{d, S}$ represent the regulation up and down capacity prices of generating units and ES devices. In (2), generating units balance the real-time load deviation trajectory $\Delta D(t)$ through the up and down balancing power. The regulation up and down requirements are provided by generating units and ES devices in (3) and (4), where $\mathbf{1}_{K}$ and $\mathbf{1}_{J}$ are $K$ and $J$-dimensional vectors of ones. The balancing power and ramping trajectories as well as the regulation capacities of generating units over $\mathcal{T}_{\tau}$ are constrained in (5)-(11), where the underlined and overlined terms respectively represent the minimum and maximum limits of the trajectories. The fourth terms in left-hand-side of (7)-(9) represent the maximum ramping capability required by the regulation up and down capacity in case they are fully deployed, where $T^{R}$ in (7)-(11) represents the maximum time duration for which the reserved regulation up and down capacity should be fully available ( 5 minutes in NERC standard [23]). In (5)-(8), $\mathbf{G}^{*}(t)$ and $\dot{\mathbf{G}}^{*}(t)$ are respectively the fixed optimal day-ahead generation and ramping trajectories of units, which are provided by the solution of the day-ahead unit commitment model in [18].

The lower and upper ES energy trajectories, shown by vectors $\underline{\mathbf{E}^{S}}(t)=\left(\underline{E_{1}^{S}}(t), \ldots, \underline{E_{J}^{S}}(t)\right)^{T}$ and $\overline{\mathbf{E}^{S}}(t)=$ $\left(\overline{E_{1}^{S}}(t), \ldots, \overline{E_{J}^{S}}(t)\right)^{T}$, are calculated in (12) over $\mathcal{T}_{\tau}$, and denote the lowest and highest energy trajectories of ES devices due to the full deployment of regulation up and down reserves, respectively, and are constrained to 
the minimum and maximum energy capacity limits of ES devices in (15) and (14). The $\boldsymbol{\eta}^{c}$ and $\boldsymbol{\eta}^{d}$ in (12) and (13) are diagonal $J \times J$ matrices of charging and discharging efficiencies. Initial values of the trajectories are enforced in (16), where $\mathbf{g}_{\tau}^{u}, \mathbf{g}_{\tau}^{d}, \mathbf{R}_{\tau}^{u, G}, \mathbf{R}_{\tau}^{d, G}, \mathbf{R}_{\tau}^{u, S}$, $\mathbf{R}_{\tau}^{d, S}$ and $\mathbf{E}_{\tau}^{S}$ are vectors of constant initial values.

\section{The Proposed Solution Method}

The model proposed in (1)-(16) is a variational problem with infinite-dimensional decision space that is computationally intractable. The common practice to solve this type of problems has been to discretize the scheduling time horizon using a finite number of sampling points and approximate the continuous-time parameter and decision trajectories with zero-order piecewise constant trajectories, as shown in Fig. 3-(a). This time-discretization approach, though, does not appropriately capture the variability and fast dynamics of real-time load in smaller time scales. In addition, discrete-time models would not appropriately capture the fast-ramping capability of generating units and ES devices to compensate the variations of load. Increasing the accuracy of discrete-time models would require increasing the number of sampling points, which would in turn increase the decision space dimensionality of the optimization problem.

In our recent works in $[18,19,20,21]$, we have developed an alternative approach for sampling the load and decision trajectories of power systems operation problems. Unlike the common approach of approximating the continuous-time load trajectories using piecewise constant curves, our approach projects the load and decision trajectories on a countable and finite-dimensional function space, as schematically shown in Fig. 3-(b). A careful choice of the function space would capture the finer information about the evolution of load in time and its ramping requirement, and appropriately deploy the available resources to supply the load and ramping requirements.

Here we intend to leverage our works in [18, 19, 21], and develop a function space-based solution method for the proposed variational problem (1)-(16). The proposed solution method is based on reducing the dimensionality of the continuous-time decision and parameter trajectories by modeling them in a finite-order function space spanned by the Bernstein polynomials. The Bernstein polynomials of degree $Q$ include $Q+1$ polynomials defined as:

$$
b_{q, Q}(t)=\left(\begin{array}{c}
Q \\
q
\end{array}\right) t^{q}(1-t)^{Q-q}, t \in[0,1],
$$

for $q=0, \ldots, Q$. Let us construct a set of basis functions

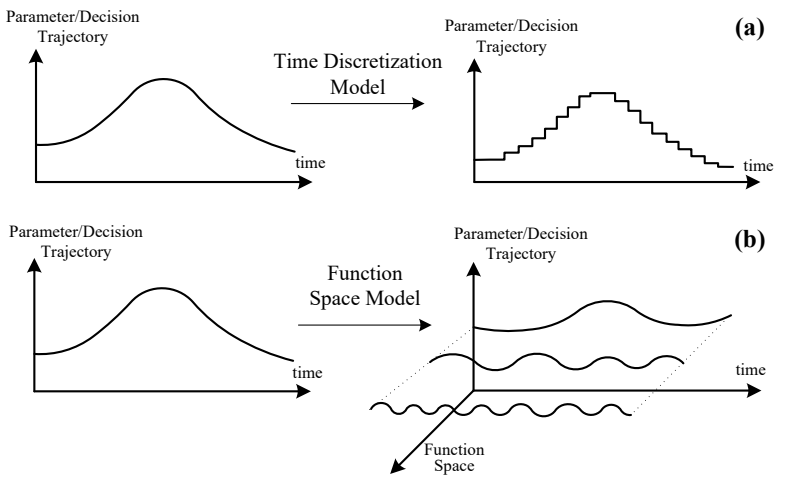

Figure 3. Trajectory modeling approaches: a) discrete-time model, b) function space model.

in each interval $n \in N$ of the receding scheduling horizon $\mathcal{T}_{\tau}$ using the Bernstein polynomials of degree $Q$. Thus, the vector of basis functions $\mathbf{e}^{(Q)}(t)=$ $\left(e_{1}^{(Q)}(t), \ldots, e_{P}^{(Q)}(t)\right)^{T}$ spanning the whole $\mathcal{T}_{\tau}$ contains $P=(Q+1) N$ functions defined as:

$$
e_{n(Q+1)+q}^{(Q)}(t)=b_{q, Q}\left(\frac{t-t_{n}}{T_{n}}\right), t \in\left[t_{n}, t_{n+1}\right),
$$

for $n=0, \ldots, N-1 ; q=0, \ldots, Q$. To reduce notation, we define $p \equiv n(Q+1)+q$, where $p$ goes from 0 to $P-1$.

In the following, we formulate different components of the proposed function space solution model.

\subsection{Modeling Balancing Power and Ramping, and Regulation Capacity Trajectories of Generating Units}

We utilize the Bernstein function space $\mathbf{e}^{(Q)}(t)$ in (18) to model the up and down balancing power trajectories of generating units as:

$$
\begin{aligned}
& \mathbf{g}^{u}(t)=\mathbf{g}^{u} \mathbf{e}^{(Q)}(t), \quad t \in \mathcal{T}_{\tau}, \\
& \mathbf{g}^{d}(t)=\mathbf{g}^{d} \mathbf{e}^{(Q)}(t), \quad t \in \mathcal{T}_{\tau},
\end{aligned}
$$

where $\mathbf{g}^{u}$ and $\mathbf{g}^{d}$ are $K \times P$ coefficients matrices of projecting the trajectories in the function space.

The time derivatives of Bernstein polynomials of degree $Q$ can be expressed as a linear combination of two Bernstein polynomials of degree $Q-1$ [24]. This property enables defining the continuous-time balancing ramping trajectories of units in the space spanned by 
Bernstein polynomials of degree $Q-1$ as follows:

$$
\begin{aligned}
\dot{\mathbf{g}}^{u}(t) & =\mathbf{g}^{u} \dot{\mathbf{e}}^{(Q)}(t)=\mathbf{g}^{u} \mathcal{M} \mathbf{e}^{(Q-1)}(t) \\
& =\dot{\mathbf{g}}^{u} \mathbf{e}^{(Q-1)}(t), \\
\dot{\mathbf{g}}^{d}(t) & =\mathbf{g}^{d} \dot{\mathbf{e}}^{(Q)}(t)=\mathbf{g}^{d} \mathcal{M} \mathbf{e}^{(Q-1)}(t) \\
& =\dot{\mathrm{g}}^{d} \mathbf{e}^{(Q-1)}(t),
\end{aligned}
$$

where $\mathcal{M}$ is a $P \times(P-N)$ matrix relating $\dot{\mathbf{e}}^{(Q)}(t)$ and $\mathbf{e}^{(Q-1)}(t)$, and $\dot{\mathbf{g}}^{u}$ and $\dot{\mathbf{g}}^{d}$ are $K \times(P-N)$ coefficients matrices of projecting the ramping trajectories in the function space, related with Bernstein coefficients of the corresponding balancing power trajectories as:

$$
\dot{\mathrm{g}}^{u}=\mathbf{g}^{u} \mathcal{M}, \quad \dot{\mathrm{g}}^{d}=\mathbf{g}^{d} \mathcal{M} .
$$

The regulation up and down trajectories of generating units are also projected into Bernstein function space spanned by $\mathbf{e}^{(Q)}(t)$ as follows:

$$
\begin{aligned}
& \mathbf{R}^{u, G}(t)=\mathbf{R}^{u, G} \mathbf{e}^{(Q)}(t), \quad t \in \mathcal{T}_{\tau}, \\
& \mathbf{R}^{d, G}(t)=\mathbf{R}^{d, G} \mathbf{e}^{(Q)}(t), \quad t \in \mathcal{T}_{\tau},
\end{aligned}
$$

where $\mathbf{R}^{u, G}$ and $\mathbf{R}^{d, G}$ are $K \times P$ coefficients matrices of projecting the trajectories in the function space. Note that, as in our work in [18], the optimal day-ahead generation and ramping trajectories of generating units, $\mathbf{G}^{*}(t)$ and $\dot{\mathbf{G}}^{*}(t)$ in (5)-(8), are modeled similar to the power and ramping trajectories above.

\subsection{Modeling Regulation Capacity Trajectories of ES Devices}

Similar to generating units, we utilize the Bernstein function space $\mathbf{e}^{(Q)}(t)$ to model the regulation up and down capacity trajectories of ES devices as:

$$
\begin{aligned}
& \mathbf{R}^{u, S}(t)=\mathbf{R}^{u, S} \mathbf{e}^{(Q)}(t), \quad t \in \mathcal{T}_{\tau}, \\
& \mathbf{R}^{d, S}(t)=\mathbf{R}^{d, S} \mathbf{e}^{(Q)}(t), \quad t \in \mathcal{T}_{\tau},
\end{aligned}
$$

where $\mathbf{R}^{u, S}$ and $\mathbf{R}^{d, S}$ are $J \times P$ coefficients matrices of projecting the trajectories in the function space.

The integral of Bernstein polynomials of degree $Q$ are linearly related to Bernstein polynomials of degree $Q+1$, suggesting that there is a $P \times(P+N)$ linear mapping, $\mathcal{N}$, relating the integral of $\mathbf{e}^{(Q)}(t)$ with $\mathbf{e}^{(Q+1)}(t)$. Using this property and integrating (12) over $t$, we derive the projection of upper ES energy trajectories in Bernstein function space as:

$$
\begin{aligned}
\overline{\mathbf{E}^{S}}(t) & =\mathbf{E}_{\tau}^{S}+\boldsymbol{\eta}^{c} \mathbf{R}^{d, S} \int_{\tau}^{t} \mathbf{e}^{(Q)}\left(t^{\prime}\right) d t^{\prime} \\
& =\mathbf{E}_{\tau}^{S}+\boldsymbol{\eta}^{c} \mathbf{R}^{d, S} \mathcal{N}^{(Q+1)}(t) \\
& =\left(\mathbf{E}_{\tau}^{S} \mathbf{1}_{P+N}^{T}+\boldsymbol{\eta}^{c} \mathbf{R}^{d, S} \mathcal{N}\right) \mathbf{e}^{(Q+1)}(t) \\
& =\overline{\mathbf{E}^{S}} \mathbf{e}^{(Q+1)}(t), \quad t \in \mathcal{T}_{\tau}
\end{aligned}
$$

where $\mathbf{E}_{\tau}^{S} \mathbf{1}_{P+N}^{T}$ in the third line is the projection of constant initial energy values vector $\mathbf{E}_{\tau}^{S}$ to the space spanned by $\mathbf{e}^{(Q+1)}(t)$, and $\overline{\mathbf{E}^{S}}$ is $J \times(P+N)$ matrix of Bernstein coefficients of upper ES energy trajectories, which is equal to:

$$
\overline{\mathbf{E}^{S}}=\mathbf{E}_{\tau}^{S} \mathbf{1}_{P+N}^{T}+\boldsymbol{\eta}^{c} \mathbf{R}^{d, S} \mathcal{N} .
$$

Similarly, we project the lower ES energy trajectories into the Bernstein function space spanned by $\mathbf{e}^{(Q+1)}(t)$ with the associated Bernstein coefficients defined as:

$$
\underline{\mathbf{E}^{S}}=\mathbf{E}_{\tau}^{S} \mathbf{1}_{P+N}^{T}-\boldsymbol{\eta}^{d^{-1}} \mathbf{R}^{u, S} \mathcal{N} .
$$

\subsection{Continuity of Decision Trajectories}

Optimality conditions of the variational problem (1)-(16) requires $C^{1}$ continuity of the decision trajectories [18]. In order to ensure $C^{1}$ continuity at the interval connection points, we impose continuity constraints on the Bernstein coefficients of adjacent intervals. For the regulation up trajectories of ES device $j$, the continuity constraints are formulated as below:

$$
\begin{aligned}
& R_{j, n(Q+1)+Q}^{u, S}=R_{j,(n+1)(Q+1)}^{u, S}, n=0, \ldots N-2, \\
& \frac{1}{T_{n}}\left(R_{j, n(Q+1)+Q}^{u, S}-R_{j, n(Q+1)+Q-1}^{u, S}\right)= \\
& \frac{1}{T_{n+1}}\left(R_{j,(n+1)(Q+1)+1}^{u, S}-R_{j,(n+1)(Q+1)}^{u, S}\right) \\
& n=0, \ldots N-2 .
\end{aligned}
$$

Similar continuity constraints are imposed on the Bernstein coefficients of the other decisions trajectories.

\subsection{Modeling Inequality Constraints}

The convex hull property of Bernstein polynomials enables us to efficiently impose the continuous-time inequality constraints (3)-(11) and (14)-(15). More specifically, the convex hull property of Bernstein polynomials states that a trajectory will never be outside of the convex hull formed by the Bernstein 
coefficients of the trajectory $[18,24]$. Therefore, the continuous-time inequality constraints (3)-(11) and (14)-(15) can be imposed respectively by constraining the associated Bernstein coefficients as follows:

$$
\begin{aligned}
& \mathbf{1}_{K}^{T} \mathbf{R}^{u, G}+\mathbf{1}_{J}^{T} \mathbf{R}^{u, S} \geq \mathbf{D}^{R^{u}} \\
& \mathbf{1}_{K}^{T} \mathbf{R}^{d, G}+\mathbf{1}_{J}^{T} \mathbf{R}^{d, S} \geq \mathbf{D}^{R^{d}} \\
& \mathbf{G}^{*}+\mathbf{g}^{u}+\mathbf{R}^{u, G} \leq \overline{\mathbf{G}} \\
& \mathbf{G}^{*}-\mathbf{g}^{d}-\mathbf{R}^{d, G} \geq \underline{\mathbf{G}} \\
& \left(\dot{\mathbf{G}}^{*}+\dot{\mathbf{g}}^{u}-\dot{\mathbf{g}}^{d}\right) \mathbf{M}+\frac{\mathbf{R}^{u, G}}{T^{R}} \leq \overline{\dot{\mathbf{G}}} \mathbf{M} \\
& \left(\dot{\mathbf{G}}^{*}+\dot{\mathbf{g}}^{u}-\dot{\mathbf{g}}^{d}\right) \mathbf{M}-\frac{\mathbf{R}^{d, G}(t)}{T^{R}} \geq \underline{\dot{\mathbf{G}}} \mathbf{M}, \\
& \mathbf{0} \leq \frac{\mathbf{R}^{u, G}}{T^{R}} \leq \overline{\dot{\mathbf{G}}} \mathbf{M}, \mathbf{0} \leq \frac{\mathbf{R}^{d, G}}{T^{R}} \leq-\underline{\dot{\mathbf{G}}} \mathbf{M}, \\
& \mathbf{0} \leq \mathbf{R}^{u, S} \leq \overline{\mathbf{R}}^{u, S}, \frac{\mathbf{R}^{u, S}}{T^{R}} \leq \overline{\mathbf{R}}^{u, S} \mathbf{M}, \\
& \mathbf{0} \leq \mathbf{R}^{d, S} \leq \overline{\mathbf{R}}^{d, S}, \frac{\mathbf{R}^{d, S}}{T^{R}} \leq \overline{\mathbf{R}}^{d, S} \mathbf{M}, \\
& \underline{\mathbf{E}} \leq \overline{\mathbf{E}^{S}} \leq \overline{\mathbf{E}}, \\
& \underline{\mathbf{E}} \leq \underline{\mathbf{E}^{S}} \leq \overline{\mathbf{E}},
\end{aligned}
$$

In (37) and (38), $\mathbf{M}$ is a $(P-N) \times P$ linear mapping that relates $\mathbf{e}^{(Q-1)}(t)$ and $\mathbf{e}^{(Q)}(t)$. In (33) and (34), $\mathbf{D}^{R^{u}}$ and $\mathbf{D}^{R^{d}}$ are $P$-dimensional vectors of Bernstein coefficients of projecting the regulation up and down requirement trajectories into the Bernstein function space spanned by $\mathbf{e}^{(Q)}(t)$ as follows:

$$
\begin{aligned}
D^{R^{u}}(t) & =\mathbf{D}^{R^{u}} \mathbf{e}^{(Q)}(t), t \in \mathcal{T}_{\tau}, \\
D^{R^{d}}(t) & =\mathbf{D}^{R^{d}} \mathbf{e}^{(Q)}(t), t \in \mathcal{T}_{\tau} .
\end{aligned}
$$

\subsection{Modeling Objective Functional and Real-time Power Balance Constraint}

It is straightforward to show that the objective functional (1) can be converted to a linear function of Bernstein coefficeints of the decision variable, as in [19]. In order to express the continuous-time power balance constraint (2) in the function space, let the real-time load deviation trajectory $\Delta D(t)$ be spanned over the Bernstein function space $\mathbf{e}^{(Q)}(t)$ as:

$$
\Delta D(t)=\Delta \mathbf{D e}^{(Q)}(t), \quad t \in \mathcal{T}_{\tau},
$$

where $\Delta \mathrm{D}$ is a $P$-dimensional row vector of Bernstein coefficients. Substituting the Bernstein models of balancing power trajectories of generating units from (19) and (20) and load trajectory from (46) in the continuous-time power balance constraint (2) and eliminating $\mathbf{e}^{(Q)}(t)$ from both sides, we derive:

$$
\Delta \mathbf{D}=\mathbf{1}_{K}^{T}\left(\mathrm{~g}^{u}-\mathbf{g}^{d}\right),
$$

which converts the continuous-time power balance constraint (2) to algebraic equations on the Bernstein coefficients.

In summary, equations (19)-(47) present the proposed function space solution method that converts the variational problem (1)-(16) into a linear programming problem with the Bernstein coefficients of decision trajectories as decision variables.

\section{Numerical Results}

The proposed model is implemented in this section on the IEEE Reliability Test System (RTS) [25]. In this study, the Bernstein polynomials of degree 3 are used for modeling the load and decision trajectories in the proposed function space solution method in Section 3. The hourly day-ahead load data of the California ISO (CAISO) for Jan. 23, 2018 [26] is scaled down to 2850MW peak load of IEEE-RTS and used to derive the Bernstein coefficients of the continuous-time day-ahead load trajectory. The day-ahead CAISO load data is then disturbed with normally distributed random error terms in order to form the real-time load, where the standard deviations of the error terms are considered to be $2 \%$ of the load at each time. The continuous-time day-ahead and real-time load trajectories are shown in Fig. 4-(a), and Fig. 4-(b) presents the continuous-time real-time load deviation from the day-ahead load. The regulation up and down requirements are considered to be equal to $1 \%$ of real-time load at each time.
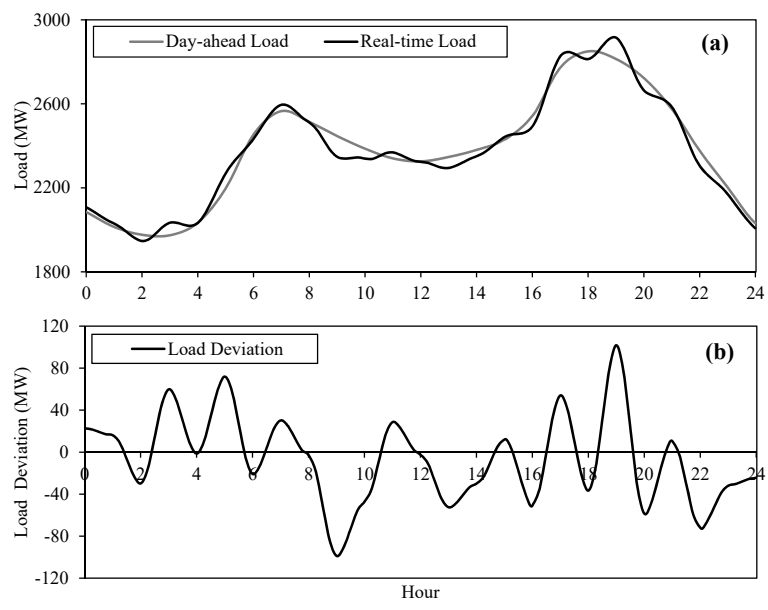

Figure 4. (a) day-ahead and real-time load trajectories (b) real-time load deviation trajectory 
Two cases are studied here. In Cases 1 and 2 , the real-time operation of IEEE-RTS are studied respectively without and with ES devices. For both cases, the optimal day-ahead generation and ramping trajectories, $\mathbf{G}^{*}(t)$ and $\dot{\mathbf{G}}^{*}(t)$, are derived from the solution of the continuous-time day-ahead unit commitment model [18], assuming that ES devices are not scheduled in day-ahead operation. The energy capacity, power rating, and ramping rate of the ES device used in Case 2 are respectively 100MWh, 50MW, and $20 \mathrm{MW} / \mathrm{min}$, the charge and discharge efficiency of the ES device are both $90 \%$, and the initial stored energy is considered to be $50 \mathrm{MWh}$. We assume that the prices of real-time balancing power and regulation up and down capacity of generating units respectively equal to $100 \%$ and $35 \%$ of their highest day-ahead energy bid (given in [25]). The regulation up and down capacity prices of the ES device are respectively $\$ 7$ and $\$ 5$ per $\mathrm{MWh}$, and the regulation scarcity cost is considered to be $\$ 250$ per MWh. Further, the original ramping capabilities of the generating units given in [25] are shrunk by the factor of 3 to better demonstrate the ES value in this study. We use 4-hour receding control horizons for each look-ahead run, repeated every 15-minutes, and update the real-time load trajectories for each of the scheduling horizons. The cases are solved using CPLEX 12.6.2 on a computer with a $4.0 \mathrm{GHz}$ i7 processor and $32 \mathrm{~GB}$ of RAM. The solution time for each of the look-ahead runs in the cases are trivial and all under one second. The numerical results are discussed below in detail.

1) Real-time operation cost: the real-time operation cost components are presented for the two cases in Table 1. In Case 1, the operation cost amounts to $\$ 20,205.3$, which includes the cost of providing energy and regulation up and down from generating units, as well as the regulation scarcity cost incurred due to the lack of available resources. In Case 2, as ES boosts the available regulation up and down capacity, it eliminates the regulation scarcity instants and reduces the real-time operation cost to $\$ 15,830.6$.

Table 1. Real-time Operation Cost Components

\begin{tabular}{c|c|c|c}
\cline { 3 - 4 } \multicolumn{2}{c|}{} & Case 1 & Case 2 \\
\hline \hline \multirow{2}{*}{$\begin{array}{c}\text { Generating } \\
\text { Units }\end{array}$} & Energy Cost (\$) & $11,023.6$ & $10,551.9$ \\
\cline { 2 - 4 } & Regulation Up Cost (\$) & $2,977.2$ & $1,696.5$ \\
\cline { 2 - 4 } & Regulation Down Cost (\$) & $2,330.1$ & $2,128.9$ \\
\hline \hline \multirow{2}{*}{$\begin{array}{c}\text { Energy } \\
\text { Storage }\end{array}$} & Regulation Up Cost (\$) & 0.0 & $1,274.8$ \\
\cline { 2 - 4 } & Regulation Down Cost (\$) & 0.0 & 178.4 \\
\hline \hline \multicolumn{2}{c}{ Regulation Scarcity Cost (\$) } & $3,874.4$ & 0.0 \\
\hline \multicolumn{2}{c}{ Total Cost (\$) } & $20,205.3$ & $15,830.6$ \\
\hline
\end{tabular}

2) Regulation trajectories of generating units and $E S$ : the continuous-time regulation up and down
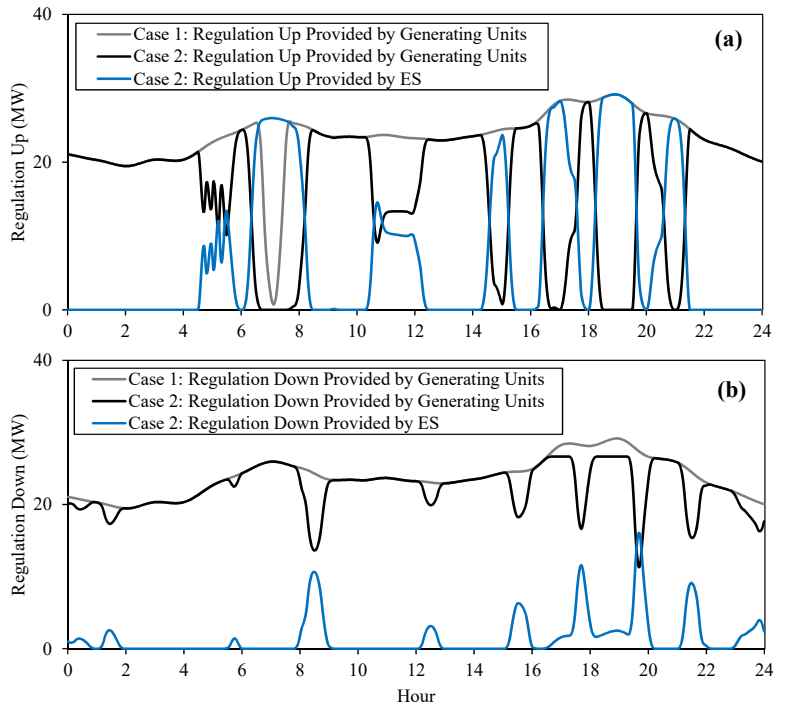

Figure 5. Regulation trajectories of generating units and the ES device in Cases 1 and 2 (a) regulation up, (b) regulation down

trajectories provided by the generating units and the ES device are shown in Figs. 5-(a) and (b) for Cases 1 and 2. In Case 1, the generating units not only supply the load deviation shown in Fig. 4-(b), but also reserve an extra capacity to meet the regulation up and down requirements as shown in Figs. 5-(a) and (b). In this case, however, the generating units fall short to cater adequate regulation up at hour 7 , where an scarcity of $15.5 \mathrm{MWh}$ arises. The high ramping capability of the ES device, which enables reaching the maximum charging/discharging capacity in less than 4 minutes, as well as its reasonable bids, render it very competitive in the regulation market. Thus, the share of generating units in providing regulation up and down in Case 2 decreases considerably as compared to Case 1 , and the ES device provides the remaining regulation up and down requirement eliminating the regulation scarcity.

3) Energy trajectories of ES: The upper and lower ES energy trajectories over the four-hour look-ahead scheduling horizon starting at hour 6, calculated from (15) and (14), are shown in Fig. 6. The upper and lower ES energy trajectories in Fig. 6 represent respectively the extreme cases of fully deploying only the regulation down and up provided by the ES device in each period. Since ES is scheduled for a considerable amount of regulation up during hours 6-8:15 (see Fig. 5-(a)), the lower energy limit in Fig. 6 starts diminishing after hour 6 and reaches zero at hour 8:15, thus ES could no longer be scheduled for the regulation up after 8:15. The upper energy limit, however, experiences smoother changes as the ES is only partially scheduled for providing regulation down during hours 6-10. 


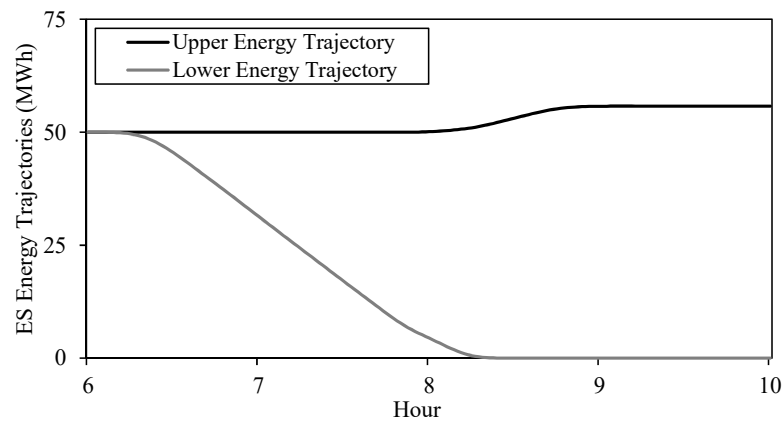

Figure 6. Upper and Lower ES Energy Trajectories

\subsection{Impact Analysis of ES Regulation Bid}

Here we aim to investigate the impact of ES regulation prices on its regulation up and down schedules and on the real-time operation cost of the system. The total scheduled regulation up and down of the ES device during the course of a day equals to the sum of areas below the regulation up and down trajectories, which is used here as an index for ES utilization in the regulation market. The real-time operation costs and the ES utilization indexes for the ES regulation down bids from $\$ 1$ per MWh to $\$ 9$ per $\mathrm{MWh}$, and the ES regulation up bids ranging from $\$ 3$ per $\mathrm{MWh}$ to $\$ 11$ per MWh, in $\$ 2$ increments, are shown in Fig. 7. Increasing the ES prices increases the operation cost and reduces the ES utilization in regulation market. The increasing trend of the operation cost and the decreasing trend of the ES utilization index continue up to the bid of $\$ 7$ and $\$ 9$ per MWh respectively for the regulation down and up, and undergo negligible changes afterwards.

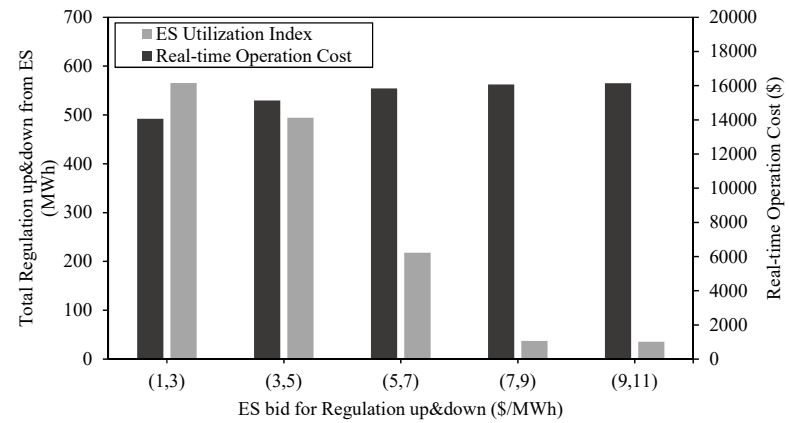

Figure 7. Impacts of ES regulation bids on system operation cost and ES regulation schedule

\section{Conclusion}

This paper proposed a continuous-time look-ahead model for scheduling the regulation capacity of generating units and ES devices in real-time operation. A function space-based solution method is then employed to reduce the decision space dimensionality of the proposed model, which utilizes the properties of Bernstein polynomials and converts the continuous-time problem into a LP problem with the Bernstein coordinates of decision trajectories as decision variables. The simulation results show that the proposed model reduces the operation cost and eliminates the regulation scarcity in real-time operation by efficiently tapping the ES flexibility to provide the regulation capacity. Future works include co-optimizing the ES regulation capacity and balancing power schedules in real-time operations, and integrating the uncertainty of load and renewable generation in the formulation.

\section{References}

[1] E. Ela, M. Milligan, and B. Kirby, "Operating reserves and variable generation," National Renewable Energy Laboratory (NREL), Golden, CO., Tech. Rep., 2011.

[2] M. R. Weimar, M. E. Mylrea, T. Levin, A. Botterud, E. O'Shaughnessy, and L. Bird, "Integrating renewable generation into grid operations: Four international experiences," Pacific Northwest National Lab.(PNNL), Richland, WA (United States), Tech. Rep., 2016.

[3] "Electric market and utility operation terminology," U.S. Department of Energy, 2011. [Online]. Available: https://www.nrel.gov/docs/fy11osti/50169.pdf

[4] J. Eyer and G. Corey, "Energy storage for the electricity grid: Benefits and market potential assessment guide," Sandia National Laboratories, pp. 69-73, 2010.

[5] M. Beaudin, H. Zareipour, A. Schellenberglabe, and W. Rosehart, "Energy storage for mitigating the variability of renewable electricity sources: An updated review," Energy for sustainable development, vol. 14, no. 4, pp. 302-314, 2010.

[6] K. Oikonomou, V. Satyal, and M. Parvania, "Energy storage in the western interconnection: Current adoption, trends and modeling challenges," in 2017 IEEE Power \& Energy Society General Meeting, 2017, pp. 1-5.

[7] A. Shahmohammadi, R. Sioshansi, A. J. Conejo, and S. Afsharnia, "The role of energy storage in mitigating ramping inefficiencies caused by variable renewable generation," Energy Conversion and Management, vol. 162, pp. 307-320, 2018.

[8] "Electric storage participation in markets operated by regional transmission organizations and independent system operators (order no. 841)," Federal Energy Regulatory Commission, 2018.

[9] D. W. Ross and S. Kim, "Dynamic economic dispatch of generation," IEEE Transactions on Power Apparatus and Systems, no. 6, pp. 2060-2068, 1980.

[10] "California iso business practice manual." [Online]. Available: http:https://www.caiso.com/rules/Pages/ BusinessPracticeManuals/Default.aspx

[11] "Business practices manual: Energy and operating reserve markets," Midcontinent ISO, 2016.

[12] L. Xie, Y. Gu, A. Eskandari, and M. Ehsani, "Fast mpc-based coordination of wind power and battery energy storage systems," Journal of Energy Engineering, vol. 138, no. 2, pp. 43-53, 2012. 
[13] D. E. Olivares, C. A. Cañizares, and M. Kazerani, "A centralized energy management system for isolated microgrids," IEEE Transactions on smart grid, vol. 5, no. 4, pp. 1864-1875, 2014.

[14] A. Lorca and X. A. Sun, "Multistage robust unit commitment with dynamic uncertainty sets and energy storage," IEEE Transactions on Power Systems, vol. 32, no. 3, pp. 1678-1688, 2017.

[15] T. Xu and N. Zhang, "Coordinated operation of concentrated solar power and wind resources for the provision of energy and reserve services," IEEE Transactions on Power Systems, vol. 32, no. 2, pp. 1260-1271, 2017.

[16] Y. Wang, Y. Dvorkin, R. Fernandez-Blanco, B. Xu, T. Qiu, and D. Kirschen, "Look-ahead bidding strategy for energy storage," IEEE Trans. Sustainable Energy, 2017.

[17] D. Dallinger, D. Krampe, and M. Wietschel, "Vehicle-to-grid regulation reserves based on a dynamic simulation of mobility behavior," IEEE Transactions on Smart Grid, vol. 2, no. 2, pp. 302-313, 2011.

[18] M. Parvania and A. Scaglione, "Unit commitment with continuous-time generation and ramping trajectory models," IEEE Transactions on Power Systems, vol. 31, no. 4, pp. 3169-3178, 2016.

[19] —, "Generation ramping valuation in day-ahead electricity markets," in 49th Hawaii International Conference on System Sciences (HICSS), 2016, pp. 2335-2344.

[20] M. Parvania and R. Khatami, "Continuous-time marginal pricing of electricity," IEEE Transactions on Power Systems, vol. 32, no. 3, pp. 1960-1969, 2017.

[21] R. Khatami, M. Parvania, and P. P. Khargonekar, "Scheduling and pricing of energy generation and storage in power systems," IEEE Trans. Power Systems, vol. 33, no. 4, pp. 4308-4322, 2018.

[22] R. Khatami, M. Heidarifar, M. Parvania, and P. Khargonekar, "Scheduling and pricing of load flexibility in power systems," IEEE Journal of Selected Topics in Signal Processing, vol. 12, no. 4, pp. 645-656, 2018.

[23] N. I. Task, "2.4 report-operating practices, procedures, and tools," 2011.

[24] P. Dierckx, "Curve and surface fitting with splines. numeric mathematics and scientific computation," Monographs on numerical analysis. Clarendon Press, Oxford Google Scholar, 1993.

[25] R. T. S. T. Force, "The ieee reliability test system-1996," IEEE Trans. Power Systems, vol. 14, no. 3, pp. 1010-1020, 1999.

[26] California ISO Open Access Same-Time Information System. [Online]. Available: http://oasis.caiso.com 